
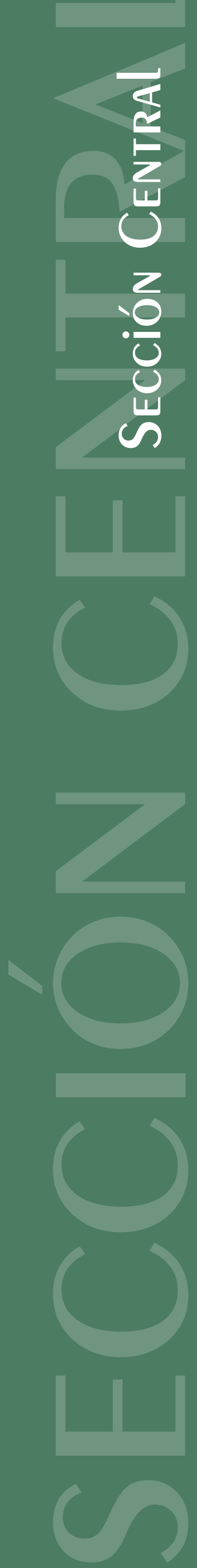


\title{
ATLETISMO DE PISTA OLÍMPICO: SEÑALES HISTÓRICAS (I)
}

\author{
TRACK ATHLETICS IN THE OLYMPICS: HISTORICAL SIGNS (I)
}

ATLETISMO DE PISTA OLÍMPICO: SEÑALES HISTÓRICAS (I)

\author{
Carlos Federico Ayala Zuluaga ${ }^{1}$ \\ Miriam Ximena Ramírez Ramírez ${ }^{2}$ \\ Cristian Alberto Ocampo Blandón ${ }^{3}$
}

Resumen

El presente es un artículo de revisión bibliográfica en el cual se presentan aspectos históricos que han sido relevantes en el atletismo de los Juegos Olímpicos modernos en el área de la pista, tanto en el escenario mundial como en el contexto latinoamericano, específicamente para Colombia. Esta historia del atletismo de pista se divide en estadios desde los Juegos de Atenas de 1896 hasta los Juegos de Tokio de 1964. Cada estadio o década presenta uno o dos atletas sobresalientes. Metodológicamente, se hizo una revisión bibliográfica basada en libros, documentos históricos, revistas especializadas impresas y digitales, consulta en bases de datos y páginas web basadas en la temática del área. Aunque se encuentran referencias para construir este tipo de artículos, hay dificultades para encontrar bibliografía especializada. La investigación evidenció gran desarrollo del atletismo de pista en el aspecto técnico y de rendimiento, especialmente en las cuatro últimas décadas. Sin embargo, es notorio que la evolución del atletismo suramericano no es relevante, aunque en Colombia se evidencian mejoras en el número de participantes y de resultados.

Palabras clave: deporte rey; historia atlética; Juegos Olímpicos modernos; récords

\section{Abstract}

This article is a bibliographical review of the relevant historical aspects of athletics in the modern Olympic Games, both worldwide and in Latin America, but more specifically in Colombia. This history of track athletics is divided by stadiums from the 1896 Athens Games to the 1964 Tokyo Games. Each stadium or decade features one or two outstanding athletes. Methodologically speaking, a bibliographic review was made based on books, historical documents, printed and digital specialized magazines, as well as research in databases and web pages focusing on the topic. The research showed a great development of track athletics in the technical and performance aspect, especially in the last four decades. However, it is evident that the evolution of South American athletics is not relevant, although in Colombia there is evidence of improvements in terms of the number of participants and results.

Keywords: sports of kings; history of athletics; modern Olympic Games; records

1 Docente asociado de la Universidad de Caldas, Grupo de Investigación Cumanday Actividad Física y Deporte. Doctor en Ciencias de la Motricidad, magíster en Educación, especialista en Procesos Pedagógicos del Entrenamiento Deportivo, licenciado en Educación Física y Recreación. Correo electrónico: federico.ayala@ucaldas.edu.co.

2 Estudiante de Licenciatura en Educación Física, Recreación y Deporte. Universidad de Caldas, integrante del Semillero de Investigación Cumanday Actividad Física y Deporte. Correo electrónico: miriam.2071521653@ucaldas.edu.co.

3 Estudiante de Licenciatura en Educación Física, Recreación y Deporte. Universidad de Caldas, integrante del Semillero de Investigación Cumanday Actividad Física y Deporte. Correo electrónico: cristian.2071424756@ucaldas.edu.co. 
0 presente é um artigo de revisão bibliográfica que apresenta aspectos históricos relevantes no atletismo dos Jogos Olímpicos modernos na área da pista, tanto no cenário mundial quanto no contexto latino-americano, especificamente colombiano. Esta história do atletismo de pista está dividida em estágios desde os Jogos de Atenas de 1896 até os Jogos de Tóquio de 1964. Cada década apresenta um ou dois atletas destacados. Metodologicamente, foi realizada uma revisão bibliográfica baseada em livros, documentos históricos, revistas especializadas impressas e digitais, pesquisa em bases de dados e sites web relacionadas com a temática da área. Ainda que seja possível encontrar referências para construir este tipo de artigos, existem dificuldades para encontrar bibliografia especializada. A pesquisa evidenciou um grande desenvolvimento do atletismo de pista no aspecto técnico e de rendimento, especialmente nas últimas quatro décadas. Porém é evidente que a evolução do atletismo na América do Sul não é relevante, ainda que na Colômbia melhorou em número de participantes e de resultados.

Palavras chave: esporte rei; história atlética; Jogos Olímpicos modernos; recordes

Fecha de recepción: 25 de julio de 2016

Fecha de aprobación: 20 de enero de 2017

Para citar este artículo:

Ayala, C., Ocampo, C. y Ramírez M. (2017). Atletismo de pista olímpico: señales históricas (I). Lúdica Pedagógica, (25), 9-20. 


\section{INTRODUCCIÓN}

Toda actividad humana tiene una historia, a través de la cual ha evolucionado de manera positiva o negativa. El atletismo es una de ellas y ha pasado por diferentes periodos, tales como el primitivo, el antiguo y el moderno. En este documento se presentan aspectos relevantes del atletismo de pista olímpico moderno, como ha evolucionado considerablemente dentro de las actividades deportivas y del atletismo en sí, como se ve desde el periodo antiguo con la realización de cinco pruebas en los Juegos Olímpicos Prehelénicos, hasta llevar a cabo 24 pruebas en varones y 23 en damas en el atletismo moderno.

Según García (1993) y Trigueros (2010), el atletismo como actividad física intensa y como deporte organizado aparece alrededor del año 3500 a. C., en el antiguo Egipto y la Mesopotamia. Así lo sugieren las evidencias encontradas en el arte rupestre, en cerámicas de bajorrelieve antiguo, pues se identifican imágenes de las primeras pruebas atléticas que consistían en carreras a pie, entre otras. Aproximadamente en el 1500 a. C., en la Grecia clásica antigua se evidencian noticias sobre actividades atléticas competitivas, basada esencialmente en carreras (Hélade).

Los eventos más conocidos y renombrados de la época antigua fueron los Juegos Olímpicos, llamados así porque se desarrollaban en la ciudad de Olimpia, en el Peloponeso. Se iniciaron aproximadamente en el 1222 a. C., continuaron con la misma dinámica y organización hasta el año 884 a. C.; aunque las primeras pruebas organizadas de las que se tiene noticia empírica se realizaron en el año 776 a. C., con olimpiadas que se realizaron exclusivamente para griegos, y en el año 228 a. C. se admitieron romanos (Casado, Díaz y Cobo, 1989; García, 1993; Jaramillo, 2004).

Los datos mejor referenciados en los anales son los aparecidos a partir del año 772 a. C. Se inició con la prueba del stadion $(197,27)$ o de velocidad, años más tarde se incluyeron las pruebas del diaulos (doble stadion, $384 \mathrm{~m}$ ), el dólico (prueba de resistencia entre $1300 \mathrm{~m}$ y $4600 \mathrm{~m}$ aproximadamente) y por último el pentatlón, que consistía en cinco pruebas (stadion, salto largo con alteras, disco, jabalina y lucha) (Casado et al., 1989; García, 1993; Hornillos, 2000; Jaramillo, 2004; Lenoir, De Clercq y Laporte, 2005; Trigueros, 2010). Estos eventos atléticos se pueden considerar como la base del deporte moderno. A partir del año $392 \mathrm{~d}$. C. los juegos se suspendieron porque se creía que ya no respondían a los ideales deportivos y competitivos para lo que fueron creados (Casado et al., 1989; García, 1993; Oliveira, 2006).

Luego, entre los siglos IV y XVII, se encuentran manifestaciones atléticas en el Reino Unido, Irlanda, las comunidades celtas (eventos denominados Lugnas Games o Tailteann Games), España, Roma (Italia), específicamente el cross-country, pruebas de carreras, el korrikolaris (corredores) -o sea una carrera a pie de larga distancia entre 10 y 20 kilómetros por relevos entre 2 corredores-, el lastorkaris (andarines) -prueba de entre 50 y 60 kilómetros, que se combinaba entre carrera y caminata-; pruebas de carreras a pie que se realizaban al estilo griego, o sea totalmente desnudos. Todos estos eventos eran practicados por personas de diversas clases sociales (Carrasco y Carrasco, 2004; Lavega y Olaso, 2007; Rodríguez, 2012). Por ahora dejamos el recorrido histórico, el cual retomaremos en la segunda parte, a partir del siglo XVII, época en la que inicia el declive de la Edad Media.

Con base en lo anterior, se presenta un panorama de aspectos, situaciones, eventos y acontecimientos en torno al atletismo de pista, específicamente el que se ha desarrollado en los diferentes Juegos Olímpicos (JJ. oo.) modernos, desde Atenas 1896 hasta Tokio 1964.

\section{ATLETISMO DE PISTA OLÍMPICO MODERNO}

Ayala, Aguirre y Agudelo (2014) nos presentan un resumen de los estadios por los cuales ha avanzado el entrenamiento deportivo de la era moderna y contemporánea. En esta primera parte exhibimos los siguientes: estadio del empirismo (1896-920) e innovaciones (Cabrera, 2010; Vrijens, 2006); estadio moderno (19201950) (Fernandes, 1981; Platonov, 2008; Vasconcelos, 2005), y estadio neomoderno (1950-1964) precientífico (sistematización del entrenamiento) (Gomes, 1980; Gomes, 2009; Hegedüs, 1973).

\section{Estadio del empirismo (1896-1920) e innovaciones}

Los JJ. oo. que comprendieron este estadio fueron: Atenas-Grecia (1896), París-Francia (1900), San Luis-EEUU (1904), Londres-Reino Unido (1908), Estocolmo-Suecia (1912), Berlín-Alemania, suspendidos por la Primera Guerra Mundial (1916) y Amberes-Bélgica (1920). 
Animado por este renacer deportivo, con los inicios del cronometraje eléctrico en 1892 en Inglaterra y la renovación de los JJ. oo., el atletismo entró en la era moderna. Precisamente este deporte fue la base para el inicio de los juegos, gracias al auspicio y fomento del barón Pierre de Coubertain, que se inspiró en los de la antigua Grecia. La primera edición tuvo lugar en Atenas en 1896 (Gispert, 2003; Matthiesen, 2007; Trigueros, 2010).

Juegos Olímpicos de Atenas (1896)

En Atenas se disputaron las carreras en pista de 100 m -exactamente 91,44 m- (Hegedüs, 2000), $400 \mathrm{~m}$ como equivalente al diaulos de la Antigüedad, que eran dos stadion (384,54 m), $110 \mathrm{~m}$ vallas, $800 \mathrm{~m}$ y $1500 \mathrm{~m}$, y la maratón con una distancia de 40000 m (Jaramillo, 2004; Matthiesen, 2007).

El estadounidense Tomas Burke fue el primer atleta en utilizar la salida baja, lo que le favoreció para la obtención del triunfo; luego se permitió la salida con apoyo de los pies en huecos en el piso (Barros y Dezem, 1978; Hegedüs, 2000; Matthiesen, 2007).

La prueba de maratón deriva su nombre de un lugar geográfico llamado así, y se propuso en el programa olímpico para recordar la gesta militar épica del soldado Filipídes de la Antigüedad. El primer ganador olímpico fue el griego Spiridon Louis, quien recorrió la distancia de $40 \mathrm{~km}$ (distancia modificada en los JJ. oo, de Londres en 1908) en 2:58:50 (Barrero, 2011; Jaramillo, 2004; Maragall y Ferrer, 1993b).

Juegos Olímpicos de París (1900)

Se realizaron paralelamente a lo que se conoce como Exposición Universal. Allí los organizadores repartieron los deportes y pruebas a lo largo de cinco meses, al igual que en los JJ. oo, de 1904 (Blanco, 2013; Casado et al., 1989). La prueba de los 200 m surgió como sustituta del stadion (192,27 m), también los $400 \mathrm{~m}$ con vallas masculino y los obstáculos masculino que fue ejecutada sobre las distancias de $2500 \mathrm{~m}$ y $4000 \mathrm{~m}$ (Jaramillo, 2004; Matthiesen, 2007).

Se destacó como el primer gran velocista de la edad moderna del olimpismo el atleta Alvin Kraezlin, al ser ganador en $60 \mathrm{~m}$ planos, en $110 \mathrm{~m}$ y $200 \mathrm{~m}$ vallas y en salto largo (Barrero, 2011).
Juegos Olímpicos de San Luis (1904)

Se incluyó en el programa olímpico la prueba combinada para hombres denominada decatlón, compuesta de 10 pruebas que son diferentes a las de la actualidad (100 yardas planas, 800 yardas marcha, 120 yardas vallas, milla (1609 m), lanzamiento de peso, de martillo y de piedra de 56 libras, y salto de pértiga, de longitud y de altura), ganada por el irlandés Thomas Francis Kiely (Maragall y Ferrer, 1993b; Matthiesen, 2007).

El atleta más destacado fue el semifondista James D. Lightbody, vencedor en los $800 \mathrm{~m}$ planos con récord olímpico, en los $1500 \mathrm{~m}$ planos con récord mundial y en los 2500 m obstáculos, además de ser medallista de plata en las cuatro millas relevos o por equipos (Maragall y Ferrer, 1993b). En la maratón se presentó un escándalo, cuando el atleta Fred Lorz (EEUU) reconoció que había sido remolcado por un automóvil, lo que le valió la descalificación de la prueba (Blanco, 2013; Casado et al., 1989).

\section{Juegos Olímpicos de Londres (1908)}

En estos JJ. 00. se construyó por primera vez un estadio, lo que permitió albergar la mayoría de deportes y pruebas (Blanco, 2013). Las carreras de relevo se incorporaron por primera vez, se llevó a cabo una prueba de velocidad combinada, en la cual los dos primeros corredores recorrían cada uno $200 \mathrm{~m}$, el tercero $400 \mathrm{~m}$ y el último 800 m. En 1912 en las olimpiadas de Estocolmo se retiró esta competición y se incluyeron las pruebas de $4 \times 100$ m y $4 \times 400$ m planos (García, 1993). Además, apareció la marcha atlética como prueba del atletismo en el programa olímpico (Jaramillo, 2004; Matthiesen, 2007).

El atleta Melvin Winfield Sheppard (EEUU) fue el más destacado al ser vencedor en los $800 \mathrm{~m}$ planos con nuevo récord mundial, en los $1500 \mathrm{~m}$ planos con récord olímpico, y el relevo olímpico (Maragall y Ferrer, 1993b). Se determinó la distancia de $42 \mathrm{~km} 195 \mathrm{~m}$ para la prueba de la maratón, la cual luego fue oficializada por la International Association of Athletics Federation (IAAF) (Barrero, 2011; Casado et al., 1989; Jaramillo, 2004; Matthiesen, 2007). En esta prueba fue descalificado el maratonista Dorando Pietri, quien fue socorrido en varias ocasiones por los jueces para terminar la prueba (Casado et al., 1989; Martínez, 1995). 
Juegos Olímpicos de Estocolmo (1912)

En estos JJ. oo. se cronometraron por primera vez las carreras y se ubicaron fotógrafos en la meta, como inicio del fotofinish; estos hechos cambiarían drásticamente la historia del atletismo de pista (Barrero, 2011; Blanco, 2013, Martínez, 1995; Rodríguez, 2012). Igualmente, se instituyó la entrega de medallas y se empezó a hacer sonar el himno nacional del país triunfador en la prueba (Casado et al., 1989; Chevalley, 2011). Se incluyeron en el programa olímpico los relevos de $4 \times 100 \mathrm{~m} \mathrm{y}$ $4 \times 400 \mathrm{~m}$ y se autorizaron las distancias de $5000 \mathrm{~m}$ y 10000 m masculino (Jaramillo, 2004; Matthiesen, 2007; Rodríguez, 2012) así como las pruebas de 3000 m obstáculos y el decatlón (Billouin, 1982). Se oficializó su disputa como se conoce hoy en día.

Ralph Cook Craig (EEUU) fue doble campeón olímpico al ser ganador de los $100 \mathrm{~m}$ y $200 \mathrm{~m}$ planos, pero la máxima figura de los JJ. oo. fue Jim Torphe (EEUU), ya que ganó las medallas de oro en el pentatlón y el decatlón, respectivamente; así logró los récords mundial y olímpico (Barrero, 2011; Casado et al., 1989; Rodríguez, 2012). A partir de entonces el programa del atletismo olímpico quedo definitivamente configurado en el plano masculino. Se batieron los récord olímpicos de los 400 m, 1500 m, 10000 m planos y la maratón, y los récords mundiales de $800 \mathrm{~m}$ y $5000 \mathrm{~m}$ planos.

\section{Juegos Olímpicos de Amberes (1920)}

La bandera olímpica fue creada en 1914, pero se izó por primera vez en estos JJ. oo. Se ha mantenido como se conoce en la actualidad, con cinco anillos que representan los continentes y los colores, pensando en que cada país tendría al menos uno de ellos en su bandera nacional; además se llevó a cabo el juramento de los atletas (Blanco, 2013; Casado et al., 1989; Chevalley, 2011; Martínez, 1995). Desde estos JJ. oo. Francia incluye corredores fondistas de los países del Magreb o del África (Matthiesen, 2007).

Aparece en el contexto olímpico el atleta de semifondo y fondo Paavo Nurmi, ganador de tres medallas doradas y una de plata (Blanco, 2013; Maragall y Ferrer, 1993b) y casi que considerado como el primer héroe olímpico (Blanco, 2013). Se batieron los récords olímpicos de los 100 m planos, $3000 \mathrm{~m}$ obstáculos, $3 \mathrm{~km}$ marcha y los récords mundiales de $110 \mathrm{~m}$ y $400 \mathrm{~m}$ vallas y el relevo de $4 \times 100 \mathrm{~m}$ planos.

\section{Estadio moderno (1920-1950)}

En este estadio encontramos los JJ. oo. modernos de París-Francia (1924), Ámsterdam-Holanda (1928), Los Ángeles-EEuU (1932), Berlín-Alemania (1936), Helsinki-Finlandia (1940), Londres-Reino Unido (1944, suspendidos por la Segunda Guerra Mundial), LondresReino Unido (1948).

\section{Juegos Olímpicos de París (1924)}

Se construyó por primera vez una Villa Olímpica, lo que contribuyó a tener más apropiación los procesos del olimpismo por parte de las delegaciones deportivas (Blanco, 2013; Chevalley, 2011; Martínez, 1995). El primer medallista dorado afroamericano fue William De-Hart, quien venció en el salto largo (Blanco, 2013). Se oficializó definitivamente la distancia de 42195 m para la prueba del maratón (Matthiesen, 2007).

En estos JJ.0o de París, Paavo Nurmi (fondista) fue conocido como "El Finlandés Volador", y se a convirtió en uno de los grandes "mitos" del atletismo mundial, pues ganó cinco medallas de oro: en $1500 \mathrm{~m}, 5000 \mathrm{~m}$, cross country individual y por equipos y los $3000 \mathrm{~m}$ por equipos planos, que se sumaron a las tres en Amberes (1920) y dos en Ámsterdam (1928). Llegó a ganar nueve medallas doradas olímpicas, además de los récords superados (Barrero, 2011; Casado et al., 1989; Martínez, 1995; Rodríguez, 2012). Los récords olímpicos que se batieron fueron en los $100 \mathrm{~m}, 200 \mathrm{~m}, 1500 \mathrm{~m}, 5000$ $\mathrm{m}$ planos y los $3000 \mathrm{~m}$ obstáculos; mientras que los récords mundiales fueron en los $400 \mathrm{~m}, 4 \times 100 \mathrm{~m}, 4 \times$ $400 \mathrm{~m}$ y los $10000 \mathrm{~m}$ planos.

\section{Juegos Olímpicos de Ámsterdam (1928)}

En estos JJ.0o. se instituyó oficialmente la llama olímpica, que simboliza la amistad y la paz que debe unir a todos los países (Casado et al., 1989; Martínez, 1995). Hubo que esperar a Ámsterdam (1928) para que la IAAF integrara al programa olímpico pruebas atléticas para mujeres (Blanco, 2013; Cortés, 2015; Perea, 2003; Robles, 2009) y verlas actuar en las pruebas de $100 \mathrm{~m}$, $4 \times 100 \mathrm{~m}$ relevos, $800 \mathrm{~m}$ y algunas de campo (salto alto y lanzamiento del disco). El retraso ha sido tanto que las carreras de $400 \mathrm{~m}$ vallas, $3000 \mathrm{~m}$ planos y la maratón solo se disputaron a partir de Los Ángeles 1984, y los 10000 m, en Seúl 1988, quedando aún pendiente para Barcelona (1992) los $10 \mathrm{~km}$ marcha atlética (DeFrantz, 1997; Jaramillo, 2004; Matthiesen, 2007). 
La estadounidense Elizabeth Robinson fue la primera campeona olímpica de atletismo, al ganar la prueba de los 100 m planos en 12,2 s (Blanco, 2013; Rueda, 2005). La marcha atlética se retiró del programa olímpico en este año, aunque en 1932 se reintegró (Matthiesen, 2007).

En estos JJ. 0o. se presentó controversia en la prueba de $800 \mathrm{~m}$ femeninos por la llegada de algunas de las atletas con signos de agotamiento extremo, lo que suscitó que no fuera prueba femenina hasta 1960 (Roma) (Blanco, 2013; Cortés, 2015; Davisse y Louveau, 1998; ). El chileno Manuel Plaza participó en la maratón y ocupó el segundo lugar, con lo que se constituyó en la primera medalla olímpica en atletismo para Suramérica (Vinker y Fontana, 1999a). En la rama masculina se batieron los récords olímpicos de $800 \mathrm{~m}, 1500 \mathrm{~m}, 10000 \mathrm{~m}$ planos y $400 \mathrm{~m}$ vallas, y los mundiales en los $3000 \mathrm{~m}$ obstáculos, $4 \times 100 \mathrm{~m}$ y $4 \times 400 \mathrm{~m}$ planos. En la rama femenina se batieron récords mundiales en los $100 \mathrm{~m}, 800 \mathrm{~m}$ y $4 \times$ 100 m planos.

Juegos Olímpicos de Los Ángeles (1932)

Se destaca en estos JJ. oo, el que por primera vez se utilizara la fotografía en la línea de meta para determinar los resultados, como gran novedad el manejo de los primeros cronómetros eléctricos, su retransmisión por radio prácticamente a todo el mundo, la utilización de un podio en tres alturas, y que los deportistas vencedores de las pruebas tuvieran el honor de ver izar su bandera y escuchar su himno (Barrero, 2011; Blanco, 2013; Casado et al., 1989; Perea, 2003).

En estos JJ. oo, se incluyó en el programa atlético la competencia de los $100 \mathrm{~m}$ vallas femenino (Matthiesen, 2007), y el primer campeón olímpico negro en los $100 \mathrm{~m}$ fue Eddie Tolan.

Mildred "Babe" Didrikson Zaharias, comenzó a destacarse en el atletismo a los 18 años, desde la velocidad hasta el lanzamiento de jabalina, participó en competencias de pentatlón y decatlón, y en 1932 fue campeona olímpica en carrera con vallas. Fue elegida en 1950 la mayor atleta de la primera mitad del siglo xx (Robles, 2009; Rodríguez, 2012).

El atleta ganador de la maratón fue el argentino Juan Carlos Zabala con 2:31:36 con nuevo récord olímpico, además de ser el campeón olímpico más joven de la especialidad, con solo 21 años de edad (Rueda, 2005;
Vinker y Fontana, 1999a). Paavo Nurmi fue descalificado por supuesto profesionalismo (Martínez, 1995). Los nuevos récords olímpicos fueron en los 200 m, $1500 \mathrm{~m}$, $5000 \mathrm{~m}, 10000 \mathrm{~m}$ planos y la maratón, y los mundiales en $100 \mathrm{~m}, 400 \mathrm{~m}, 800 \mathrm{~m}, 4 \times 100 \mathrm{~m}$ y $4 \times 400 \mathrm{~m}$ planos y el decatlón. En la rama femenina solo se batieron récords mundiales en $100 \mathrm{~m}$ y $4 \times 100 \mathrm{~m}$ planos, y en $80 \mathrm{~m}$ vallas.

Juegos Olímpicos de Berlín (1936)

En estos JJ. 0o, comenzó la tradición de la antorcha olímpica que cada cuatro años se enciende en Olimpia y es llevada en relevo hasta la sede de los juegos y debe permanecer encendida hasta el último día de competencias, ritual que se instituyó en Berlín (Chevalley, 2011; Martínez, 1995; Rueda, 2005). Las competencias de atletismo se retransmitieron por televisión por primera vez; allí se mostraban las competiciones de todos los atletas, aunque solo se hizo para el territorio alemán (Blanco, 2013; Casado et al. 1989; Rueda, 2005). En los JJ. oo. de Berlín se definió la reglamentación para la realización de las pruebas de vallas, lo que favoreció el desarrollo del entrenamiento técnico (Jaramillo, 2004).

El atleta más sobresaliente fue el estadounidense Jesse Owens, quien ganó 4 medallas de oro: en los $100 \mathrm{~m}$ y $200 \mathrm{~m}$ planos, salto largo y el relevo de $4 \times 100 \mathrm{~m}$ planos; en estas tres últimas logró récord mundial, lo que despertó la indignación de Hitler (Barrero, 2011; Casado et al., 1989; Hegedüs, 2000; Rodríguez, 2012). Se obtuvieron sendos récords olímpicos masculinos en $5000 \mathrm{~m}$ planos, en la maratón y los $50 \mathrm{~km}$ marcha; a nivel de récords mundiales se obtuvieron los de $200 \mathrm{~m}, 1500$ m y $4 \times 100$ m planos, 3000 m obstáculos y el decatlón. Los récords olímpicos femeninos nuevos fueron en los 80 m vallas, con una marca de 11,7 s en un triple empate de las atletas.

\section{Juegos Olímpicos de Londres (1948)}

Apareció en la órbita olímpica el atleta Emil Zatopek (checo), quien fue el vencedor en las pruebas de $5000 \mathrm{~m}$ y $10000 \mathrm{~m}$ planos. Se destacó por ser el primer atleta en bajar de 30 min en los 10000 m planos, con un tiempo de 29:59, lo que le valió el apodo de la "Locomotora Humana”, (Casado et al., 1989; Martínez, 1995; Matthiesen, 2007; Rodríguez, 2012). Se llevó a cabo por primera vez la prueba de $200 \mathrm{~m}$ planos para mujeres (Matthiesen, 2007), con una participación destacada de la atleta holandesa Fanny Blankers-Koen (apodada la "Holandesa 
Voladora"), ganadora de las pruebas de 100 m y $200 \mathrm{~m}$ planos, $80 \mathrm{~m}$ con vallas (con nuevo récord olímpico) y el relevo $4 \times 100 \mathrm{~m}$ planos (Barrero, 2011; Blanco, 2013; Casado et al., 1989; Jaramillo, 2004; Maragall y Ferrer, 1993b; Robles, 2009).

Bob Mathias (EEUU) fue el campeón olímpico más joven del decatlón -algunos historiadores dicen que tenía 17 años y otros, 18-, revalidando así su título en la olimpiada de Helsinki 1952 (Billouin, 1982; Maragall y Ferrer, 1993b). Delfo Cabrera, fondista argentino, resultó vencedor en la maratón olímpica, repitiendo la hazaña de su antecesor Zabala (1932). Lloyd La Beach, atleta panameño de velocidad, con 22 años ocupó el tercer lugar en la final de los $100 \mathrm{~m}$ y $200 \mathrm{~m}$ planos olímpicos (Quercetani, 1999; Vinker y Fontana, 1999a). En varones solo hubo récords olímpicos en los $100 \mathrm{~m}$, $400 \mathrm{~m}, 800 \mathrm{~m}, 5000 \mathrm{~m}, 10000 \mathrm{~m}$ planos y en los 110 $\mathrm{m}$ y $400 \mathrm{~m}$ vallas. En las damas se presentaron récords olímpicos en 100 m y 200 m planos y en los 80 m vallas.

\section{Estadio neomoderno-precientífico o sistematización del entrenamiento (1950-1964)}

Se realizaron en este estadio los JJ. oo. De HelsinkiFinlandia (1952), Melbourne-Australia (1956), RomaItalia (1960) y Tokio-Japón (1964).

Juegos Olímpicos de Helsinki (1952)

Por primera vez irrumpió en el contexto del deporte olímpico la Unión de Repúblicas Socialista Soviéticas (URSS), lo cual contribuyo considerablemente al desarrollo y la confrontación competitiva del deporte moderno, específicamente entre países socialistas y capitalistas (Casado et al., 1989; Rueda, 2005). El atleta más sobresaliente fue Emil Zatopek, quien realizó la hazaña de ganar tres medallas doradas al vencer en los 5000 m, 10000 m y la maratón en el tiempo de dos días seguidos, además de marcar nuevos récords mundiales (Barrero, 2011; Blanco, 2013; Casado et al., 1989; Maragall y Ferrer, 1993b). La marcha atlética para hombres quedó definida en las distancias de 20 km y 50 km (Matthiesen, 2007).

En la maratón ganada por Emil Zatopek, el argentino Reinaldo Gorno ocupó la segunda plaza (Quercetani, 1999; Vinker y Fontana, 1999a).
En estos JJ. 00, se destaca que los varones batieron récords olímpicos en la mayoría de pruebas de pista $(200 \mathrm{~m}$, $400 \mathrm{~m}, 800 \mathrm{~m}, 1500 \mathrm{~m}, 5000 \mathrm{~m}$ y $10000 \mathrm{~m}$ planos, $110 \mathrm{~m}$ y $400 \mathrm{~m}$ vallas); se batieron récords mundiales en los 3000 m obstáculos y los $4 \times 400$ m planos. Las damas solo mejoraron los récords mundiales en $100 \mathrm{~m}$ y $4 \times$ $100 \mathrm{~m}$ planos, y en los $80 \mathrm{~m}$ vallas.

\section{Juegos Olímpicos de Melbourne (1956)}

Por primera vez se albergan tanto hombres como mujeres en una Villa Olímpica (Chevalley, 2011).

Los atletas destacados fueron: el velocista B. J. Morrow, ganador de medallas doradas en los $100 \mathrm{~m}, 200 \mathrm{~m}$ y el relevo 4 x 100m planos, este último con record olímpico (Casado et al. 1989; Maragall y Ferrer, 1993b), y el soviético Valdimir Kuts, quien venció en los $5000 \mathrm{~m}$ y $10000 \mathrm{~m}$ planos con récords mundiales en ambas pruebas (Maragall y Ferrer, 1993b).

En estos JJ. oo. se destacó la australiana Betty Cuthbert, quien con 18 años de edad se convirtió en triple medallista dorada en las pruebas de $100 \mathrm{~m}, 200 \mathrm{~m}$ (récord olímpico) y el relevo 4 × $100 \mathrm{~m}$ (Barrero, 2011).

Igual que en los anteriores JJ. 00, los varones batieron la mayoría de récords olímpicos en casi todas las pruebas del atletismo, específicamente en pista fueron en $800 \mathrm{~m}, 1500 \mathrm{~m}, 5000 \mathrm{~m}$ y $10000 \mathrm{~m}$ planos, $110 \mathrm{~m} \mathrm{y}$ $400 \mathrm{~m}$ vallas, $3000 \mathrm{~m}$ obstáculos y en decatlón. El único record mundial batido fue en los $4 \times 100 \mathrm{~m}$ planos. En el género femenino se batieron los récords olímpicos de los $200 \mathrm{~m}$ planos y $80 \mathrm{~m}$ vallas, los $4 \times 100 \mathrm{~m}$ planos como récord mundial.

Juegos Olímpicos de Roma (1960)

Fueron considerados los primeros JJ. oo. de la modernidad; y el Comité Olímpico Internacional (CoI) autorizó su transmisión en directo por televisión a todo el mundo (Martínez, 1995). Se marcó un nuevo récord olímpico en los $400 \mathrm{~m}$ vallas masculino, cuando el atleta Glenn Davis (EEUU) superó la barrera de $50 \mathrm{~s}$ al realizar 49,3 s (Hegedüs, 2011). Abebe Bikila (Etiopía) fue el primer africano negro en ganar la maratón olímpica; corrió sin zapatos-tenis y repitió la victoria en esta misma prueba en los Jj. 0o. de Tokio, además de batir el récord mundial (Barrero, 2011; Blanco, 2013; Casado et al. 1989; Maragall y Ferrer, 1993a; Maragall y Ferrer, 1993b). 
El estadounidense Rafer Johnson fue el primer decatlonista de raza negra ganador de medalla dorada olímpica, con solo 20 años de edad (Billouin, 1982).

La prueba de $800 \mathrm{~m}$ femeninas se incluyó por primera vez en el programa olímpico (Matthiesen, 2007). La estadounidense Wilma Rudolph, denominada la "Gacela Negra”, se destacó al adjudicarse tres medallas de oro en atletismo tras ganar los $100 \mathrm{~m}$ planos, los $200 \mathrm{~m}$ planos con récord olímpico incluido, y el relevo de $4 \times 100 \mathrm{~m}$ con récord mundial (Maragall y Ferrer, 1993a; Maragall y Ferrer, 1993b; Robles, 2009). Se batieron los récords olímpicos de varones en $100 \mathrm{~m}, 800 \mathrm{~m}$ y $10000 \mathrm{~m}$ planos, $400 \mathrm{~m}$ vallas, $3000 \mathrm{~m}$ obstáculos y el decatlón; y los mundiales en $200 \mathrm{~m}, 400 \mathrm{~m}, 1500 \mathrm{~m}, 4 \times 100 \mathrm{~m}, 4 \times 400$ $\mathrm{m}$ planos y la maratón. En las competencias femeninas se marcó un nuevo récord mundial en los $800 \mathrm{~m}$ planos.

Juegos Olímpicos de Tokio (1964)

Considerados como los primeros JJ. oo. de la contemporaneidad por las instalaciones, los escenarios y elementos, y las nuevas técnicas deportivas (Maragall y Ferrer, 1993a). Por primera vez se compitió en una pista de atletismo compuesta de ocho carriles (Martínez, 1995). Las mujeres participaron en doce pruebas atléticas (Cortés, 2015); se incorporaron al programa olímpico los $200 \mathrm{~m}$ planos femenino y la prueba combinada del pentatlón femenino (impulso de la bala, salto largo, $100 \mathrm{~m}$ planos, salto alto y lanzamiento de jabalina), luego en Los Ángeles (1984) se pasó al Heptatlón o siete pruebas (Matthiesen, 2007), también se incluyeron los $400 \mathrm{~m}$ y $800 \mathrm{~m}$ planos. En estos juegos se mejoraron varios récords olímpicos en el atletismo por parte del género femenino (Casado et al., 1989; Robles, 2009, Rueda, 2000).

El atleta estadounidense Robert Hayes fue el ganador de los $100 \mathrm{~m}$ planos y el primero en hacer 10 segundos en esta prueba en unos JJ. 0o., así impuso un nuevo récord; además, ganó los $4 \times 100 \mathrm{~m}$ planos, a partir de este momento se considera como uno de los hombres más rápidos que ha tenido el atletismo mundial (Casado et al., 1989; Jaramillo, 2004; Maragall y Ferrer, 1993a; Maragall y Ferrer, 1993b). Se mejoraron los récords olímpicos varones en los $200 \mathrm{~m}, 800 \mathrm{~m}$ y $10000 \mathrm{~m}$ planos, $3000 \mathrm{~m}$ obstáculos y en los $20 \mathrm{~km}$ y $50 \mathrm{~km}$ marcha; se batieron los récords mundiales en $100 \mathrm{~m}, 4 \times 100 \mathrm{~m}$ y $4 \times 400 \mathrm{~m}$ planos y en la maratón. Las damas mejoraron los récords olímpicos de 200 m y 400 m planos; y los mundiales en los $4 \times 100 \mathrm{~m}$ y $800 \mathrm{~m}$ planos y el pentatlón.

\section{COLOMBIA EN EL ATLETISMO DE PISTA OLÍMPICO}

La historia documentada del atletismo colombiano -por cierto muy escasa- se rastrea desde los primeros años de la segunda década del siglo xx. A partir de estas fechas, y según lo analizado, se podría determinar que su desarrollo ha sido lento, sin desconocer la presencia de atletas destacados en el contexto atlético mundial, lo que los hace de cierta manera especiales (Villafrades, 2005), además si tenemos en cuenta los procesos que se han llevado a cabo en Colombia para el fomento y desarrollo del atletismo en sí. En este recorrido histórico colombiano de la primera parte de este artículo, se presentan datos del atletismo de pista olímpico desde el estadio del empirismo hasta el estadio científico.

\section{Estadio del empirismo (1896-1920) e innovaciones}

En este estadio no se presentó ninguna representación del atletismo colombiano en el contexto de los JJ. oo, modernos.

\section{Estadio moderno (1920-1950)}

La primera representación del atletismo colombiano en unas olimpiadas fue en Los Ángeles (1932) con el atleta Jorge Perry Villate, quien participó en la prueba de la maratón sin la fortuna de terminar a causa de un desmayo. Su participación fue posible gracias al apoyo del mismo coI, además fue el abanderado de Colombia (García, 1993; LA84 Foundation, 2010; Mulkeen, 2015; Rueda, 2005). En Berlín (1936) participaron José Domingo Sánchez (abanderado colombiano) y Campo Elías Gutiérrez (velocistas), Pedro Emilio Torres (semifondista), los fondistas Hernando Navarrete y Hugo Acosta, el cual se inscribió pero no participó por enfermedad, y Pedro del Veccio (decatlonista y saltador); se destaca la participación de Navarrete al ocupar el puesto número 18 en los 5000 m planos (Galvis, 2015a; García, 1993; Rueda, 2005).

Jaime Aparicio y Mario Rosas fueron los dos únicos colombianos que compitieron en los JJ. 0o. de Londres (1948), pero sin figuración relevante, aunque es de anotar que Aparicio solo tenía dos años de iniciar en el atletismo (Galvis, 2015c; García, 1993; Rueda, 2005). Este atleta repitió participación en los JJ. oo, de Melbourne (1956); además, fue uno de los primeros colombianos en lograr con sus actuaciones un verdadero impacto a nivel continental. Fue especialista en $400 \mathrm{~m}$ con vallas y 
ganó medallas de oro en diferentes eventos deportivos, como Juegos Bolivarianos, Centroamericanos y del Caribe, Juegos Panamericanos y Campeonatos Sudamericanos (Galvis, 2015c; García, 1993; Mulkeen, 2015).

Estadio neomoderno (1950-1964)-precientífico (sistematización del entrenamiento)

En 1956, en los JJ. 00, de Melbourne, participaron los atletas Jaime Aparicio ( $400 \mathrm{~m}$ planos y $400 \mathrm{~m}$ con vallas) y Guillermo Zapata y Alfonso Núñez; Rubén Guevara se inscribió pero no participó (García, 1993; Rueda, 2005). Aparicio clasificó a las semifinales de los $400 \mathrm{~m}$ vallas, superando la marca suramericana con un tiempo de $51,8 \mathrm{~s}$, aunque no le alcanzó para llegar a la final (Galvis, 2015c). Además, este atleta tuvo el orgullo de ser el abanderado de la delegación colombiana.

Francisco Gutiérrez, Pedro Grajales, José Gregorio Neira y Álvaro Mejía fueron los integrantes del contingente colombiano que compitió en Tokio (1964). En este grupo de atletas, Mejía surgió como el corredor de fondo más importante del país: obtuvo numerosas medallas de oro en diversos campeonatos continentales; en 1971 fue el primer suramericano en ganar la maratón de Boston (García, 1993; Mulkeen, 2015, Ramírez, 1997). El atleta Pedro Grajales fue el primer colombiano que pudo pasar de la primera eliminatoria en los 400 m planos, logró un meritorio 47,2 s, marca nacional que duro veintidós años (Galvis, 2015b; Rueda, 2005).

\section{Estadio científico (1964-1984)}

Los atletas colombianos participantes en los JJ. oo, de México (1968) fueron Pedro Grajales y Álvaro Mejía, como repitentes, y por primera vez Jimmy Sierra y Hernando Arrechea (García, 1993). Mejía terminó décimo en los 10 000m planos; esta se constituyó en la mejor actuación de un colombiano en JJ. oo, hasta ese momento (Mulkeen, 2015; Ramírez, 1997; Rueda, 2005). En Múnich (1972) participaron por primera vez atletas femeninas colombianas; ellas fueron Juana Mosquera (100 m y 200 m planos), que pasó a la segunda ronda eliminatoria, y Elsy Rivas (400 m planos), eliminada en la primera ronda. Los atletas Víctor Manuel Mora García y Domingo Tibaduiza Reyes (10 000 m planos), también tuvieron su primera incursión olímpica, este último ocupó la plaza número doce en la final de los $10.000 \mathrm{~m}$ planos; repitieron JJ. oo, Álvaro Mejía, Jimmy Sierra y Hernando Arrechea (García, 1993; Rueda, 2000).
Domingo Tibaduiza fue otro fondista colombiano de enorme talento. Fue campeón de maratones como la de Auckland (Nueva Zelanda) en 1981 y la maratón de Berlín en 1982, y segundo en la Copa de Naciones de Maratón en Montreal (1981); en 1983 fue campeón en la maratón de Manila, segundo en la de Estocolmo y octavo en la de Nueva York. También cuatro veces ocupó el podio en Juegos Panamericanos y sus registros nacionales se han mantenido intactos en los $5000 \mathrm{~m}$ (13:20.07) y 10000 m (27:53.02) logrados en Zúrich y Viena, respectivamente, en 1978 (Mulkeen, 2015; Ramírez, 1997). Además fue reconocido como el primer atleta profesional de Colombia e incluso de Latinoamérica (Galvis, 2016).

En los JJ. oo, de Montreal (1976) participaron como repitentes los atletas Víctor Manuel Mora García (10 000 m planos) y Domingo Tibaduiza Reyes (5000 m y 10000 m planos); por primera vez participaron Jesús A. Villegas Cándelo (400 m vallas), Jairo Cubillos Ramírez y Rafael Mora Zamora (maratón), Rafael Vega Hernández y Ernesto Alfaro Bermúdez (20 km marcha) (García, 1993). El atleta Domingo Tibaduiza ocupó el octavo puesto en la final de los $5000 \mathrm{~m}$ planos y el décimo en los $10000 \mathrm{~m}$ planos; y Víctor Mora ocupó el undécimo puesto. Fue una excelente representación de estos dos atletas.

En Moscú (1980), Domingo Tibaduiza Reyes (10 000 m planos y Maratón) y Ernesto Alfaro Bermúdez (20 km y $50 \mathrm{~km}$ marcha) repitieron olimpiadas; Luis Francisco Barbosa (maratón) y Enrique Peña (20 km y 50 km marcha) participaron por primera vez (García, 1993). No se obtuvieron resultados relevantes de los colombianos en estas justas.

A los JJ. oo, de Los Ángeles (1984) asistieron Domingo Tibaduiza Reyes (10 000 m planos y maratón), Manuel Ramírez Caicedo (200 m y 400 m planos) y José Querubín Moreno Moreno (20 km y 50 km marcha). Tibaduiza participó de manera consecutiva en cuatro olimpiadas (Múnich 1972, Montreal 1976, Moscú 1980 y Los Ángeles 1984). Héctor José Moreno Moreno (20 km marcha) y Francisco Vargas (20 km marcha) compitieron por primera vez (García, 1993). Querubín Moreno ocupo la novena posición y Héctor Moreno la duodécima en los 20 km marcha olímpica (Ramírez, 1997). Los colombianos se destacaron en la marcha atlética en los últimos años, ya que en 1983 Querubín Moreno fue campeón Iberoamericano en los $20 \mathrm{~km}$ marcha, 
en 1987, Querubín y Héctor Moreno se convirtieron en los primeros marchistas colombianos en ubicarse entre los diez mejores del mundo en el Campeonato Mundial de la IAAF (Mulkeen, 2015; Ramírez, 1997; Vinker y Fontana, 1999b).

\section{CONCLUSIONES}

Es importante pensar que aunque haya referencias bibliográficas de diversas índoles, para la construcción este tipo de artículos con temas específicos y especializados, en nuestro contexto se presentan bastantes dificultades para encontrar bibliografía altamente relacionada con el atletismo mundial y colombiano. Esto nos lleva a pensar en promover y convocar a los amantes e investigadores de este deporte a referenciar los acontecimientos de manera empírico-científica.

Se evidencia, según los datos referenciados, que en cada estadio o década se presentan uno o dos atletas sobresalientes, específicamente en las pruebas de velocidad de 100 m, $200 \mathrm{~m}$ y 4 x 100 m planos, y en los $5000 \mathrm{~m}$ y 10000 m planos, y la maratón.

La evolución del atletismo suramericano, al compararlo con el contexto mundial, no es significativo y relevante.

En una segunda parte, abordaremos la historia del atletismo de pista olímpico desde el estadio científico hasta el de sistematización contemporánea.

\section{REFERENCIAS}

Ayala, C., Aguirre, H. y Agudelo, A. (2014). Aspectos históricos de la teoría y metodología del entrenamiento deportivo. (Primera parte-estadios pre-helénicos hasta el Renacimiento). Revista Ímpetus, 8(1), 121-128.

Barrero, A. (2011). Los héroes de los juegos olímpicos modernos y la evolución del equipamiento deportivo en el atletismo. Wanceulen: Educación Física Digital, 8, 3.

Barros, N. y Dezem, R. (1978). O Atletismo. São Paulo: A Gazeta Maçônica.

Billouin, A. (1982). Atletismo II. Saltos, lanzamientos, decatlón y pentatlón. Barcelona: Editorial Hispano-Europea. S.A.

Blanco, A. (2013). Los juegos olímpicos: un paseo por la historia. Lección inaugural 2013/2014.
Cabrera, E. (2010). Evolución histórica del entrenamiento deportivo. R. Cejuela, J. Cortell, J. Chinchilla y J. Pérez. Nuevas Tendencias en Entrenamiento Deportivo. Alicante. Imprenta Gamma.

Carrasco, B. y Carrasco, B. (2004). Juegos y deportes tradicionales. Instituto Nacional de Educación Física - INEF. Editorial Universidad Politécnica de Madrid.

Casado, J., Díaz del Cueto, M. y Cobo, R. (1989). Educación Física. (2. ${ }^{\mathrm{a}}$ Ed.) Madrid: Editorial Pila Teleña.

Chevalley, A. (2011). Le Musée Olympique. ¿Qué sabes de los Juegos Olímpicos? (3. ${ }^{a}$ ed.). Lausana: Museo Olímpico de Lausana.

Cortés, J. (2015). Mujeres en los Juegos Olímpicos, de Atenas (1896) a Berlín (1936): género, cuerpo y poder. Universidad de Oviedo. Master Universitario Género y Diversidad (tesis de maestría).

Davisse, A. y Louveau, C. (1998). Sports, école, société: la différence des sexes: féminin, masculin et activités sportives. Editorial L'Harmattan.

De Frantz, A. (1997, juin-juillet). L'évolution du rôle de la femme aux Jeux Olympiques. Revue Olympique, 15, 18-21.

Fernandes, J. (1981). O Treinamento Desportivo. Procedimentos, organização e métodos. São Paulo: Editora Pedagógica e Universitária.

Galvis, A. (2015a). Cecilia Navarrete: toda una vida en el deporte. Federación Colombiana de Atletismo. Recuperado de http://www.fecodatle.org/cecilia-navarretetoda-una-vida-en-el-deporte/.

Galvis, A. (2015b). Pedro Grajales: querer es poder. Federación Colombiana de Atletismo. Recuperado de http:// www.fecodatle.org/pedro-grajales-querer-es-poder/.

Galvis, A. (2015c). El primer "fuera de serie", Jaime Aparicio Rodewaldt. Federación Colombiana de Atletismo. Recuperado de http://www.fecodatle.org/el-primerfuera-de-serie-jaime-aparicio-rodewaldt/.

Galvis, A. (2016). Hace 35 años se acabó el amateurismo. Federación Colombiana de Atletismo. Recuperado de http://www.fecodatle.org/hace-35-anos-se-acabo-elamateurismo/.

García, J. (1993). De cero a gesto. Proceso metodológico para la enseñanza de las diferentes pruebas atléticas (3. ${ }^{\mathrm{a}}$ ed.). Bogotá: Fecon.

Gispert, C. (2003). Manual de educación física y deportes: técnicas y actividades prácticas. Barcelona: Océano.

Gomes, A. (2009). Treinamento Desportivo: estruturação e periodização. (2..$^{\mathrm{a}}$ ed.). Porto Alegre: Artmed. 
Gomes, M. (1980). Metodologia Científica do Treinamento Desportivo. São Paulo: Ibrasa. Instituição Brasileira de Difusão Cultural.

Hegedüs, J de. (1973). Teoría general y especial del entrenamiento deportivo. Buenos Aires: Stadium.

Hegedüs, J de. (2000). Historia de las carreras de velocidad: los 100 metros llanos. Revista Digital -EFDeportes, 5(21). Recuperado de http://www.efdeportes. com/efd21b/100m.htm.

Hegedüs, J de. (2010). Historia de los récords mundiales de los 100 metros del atletismo damas. Revista Digital-EFDeportes, 15(143). Recuperado de http://www. efdeportes.com/efd143/records-mundiales-de-los100-metros-damas.htm.

Hegedüs, J de. (2011). Historia de los récords mundiales de los 400 metros con vallas, varones. Revista DigitalEFDeportes, 16(159). Recuperado de file://C:/Users/ user/Downloads/DialnetHistoriaDeLosRecordsMundialesDeLos400MetrosConVall-4684713.pdf.

Hornillos, I. (2000). Atletismo. Barcelona: INDE.

Jaramillo, C. (2004). Atletismo básico. Fundamentos de pista y campo (2. e ed.). Armenia: Kínesis.

Lavega, P. y Olaso, S. (2007). Mil juegos y deportes populares y tradicionales. Barcelona: Paidotribo.

LA84 Foundation. (2010). Colombia and Olympism. Recuperado de http://library.la84.org/OlympicInformationCenter/OlympicReview/1980/ore158/ORE158q.pdf.

Lenoir, M., De Clercq, D. y Laporte, W. (2005). The "how" and "why" of the ancient Greek long jump with weights: a five-fold symmetric jump in a row? J Sports Sci, 23(10): 1033-1043.

Maragall, P. y Ferrer, C. (1993a). Gran historia de las olimpiadas y de los deportes. De Tokio 1964 a Seúl 1988 (tomo 2). Barcelona: Difusora Internacional.

Maragall, P. y Ferrer, C. (1993b). Gran Historia de las Olimpiadas y de los Deportes. Barcelona '92/Biografías olímpicas (tomo 3). Barcelona: Difusora Internacional.

Martínez, E. (1995). Reseña histórica y cronológica de los Juegos Olímpicos (2. ${ }^{a}$ ed.). Bogotá: Instituto Colombiano de la Juventud y el Deporte (Coldeportes).

Matthiesen, S. (2007). Atletismo. Teoria e prática. Educação Física no Ensino Superior. Rio de Janeiro: Guanabara Koogan.

Mulkeen, J. (2015). La orgullosa historia del atletismo Colombiano. IAAF. World Youth Championships. Recuperado de http://cali2015.co/la-orgullosa-historia-delatletismo-colombiano/.
Oliveira. M. (2006). Atletismo escolar. Proposta de ensino na educação infantil. Rio de Janeiro: Sprint.

Perea, T. (2003). Reglamento de atletismo. Bogotá: Panamericana.

Platonov, V. (2008). Tratado Geral de Treinamento Desportivo. São Paulo: Phorte.

Quercetani, R. (1999). Sudamérica 80. Mis recuerdos sudamericanos. En L. Vinker y E. Fontana (eds.). Libro de oro del Atletismo Suramericano. 80 Años (pp. 77-84). Bogotá.

Ramírez, G. (1997). Momentos históricos del atletismo colombiano. En: Atletismo Colombiano. Revista Oficial de la Federación Colombiana de Atletismo. 6(6), 12-19. Bogotá: Ruecolor.

Robles, J. (2009). Evolución del papel de la mujer en el movimiento olímpico internacional. En: I Congreso Virtual sobre Historia de las Mujeres (p. 37).

Rodríguez, E. (2012). Libro I de los juegos Olímpicos. Buenos Aires: Alarco.

Rueda, J. (2000). Ximena Restrepo: la mejor de Colombia. Biblioteca Virtual del Banco de la República. Recuperado de http://www.banrepcultural.org/blaavirtual/ revistas/credencial/julio2000/127ximena.htm.

Rueda, J. (2005). Citius, Altius, Fortius. Colombia Olímpica Hazañas y desengaños en la historia deportiva del país. Biblioteca Virtual del Banco de la República. Recuperado el 24 de junio de 2016 de http://www.banrepcultural.org/node/32617.

Trigueros, J. (2010). Historia del atletismo. Recuperado de: http://www.deportes.info/historia-del-atletismo/.

Vasconcelos, R. (2005). Planificación y organización del entrenamiento deportivo. Barcelona: Paidotribo.

Villafrades, F. (2005). Atletismo formativo. Bucaramanga: Editorial Limitada.

Vinker, L. y Fontana, E. (1999a). El podio olímpico. En L. Vinker y E. Fontana (eds.). Libro de oro del atletismo suramericano. 80 años (pp.11-16). Bogotá.

Vinker, L. y Fontana. E. (1999b). Estadísticas. Las series mundiales. En L. Vinker y E. Fontana (eds.). Libro de oro del atletismo suramericano. 80 años (pp. 41-50). Bogotá.

Vrijens, J. (2006). Bases del entrenamiento deportivo. Barcelona: Paidotribo. 